\title{
An Environment to Support the Evaluation of the Business Value of Information Systems
}

\author{
$V$. Serafeimidis and S. Smithson \\ Department of Information Systems, London School of Economics, \\ London WC2A 2AE, UK
}

Tel01719557655Fax01719557385e-mails.smithson@lse.ac.uk

\begin{abstract}
This paper discusses the nature and the support needs for the evaluation of information systems based on current and future demands. It describes an environment to support evaluation on two levels. At the conceptual level, a conceptual framework and a set of methodological guidelines are proposed. At the operational level, we propose a groupware solution complemented by a case-based reasoning component.
\end{abstract}

\section{INTRODUCTION}

Over the years, the role of information systems (IS) in organizations has changed. They are no longer solely used to support or automate low-level or peripheral organizational functions. Instead, they are increasingly seen as a central feature of the firm's mainstream products or services, or of their delivery system. This may involve radical transformations in products, organizational structures, work-roles, and patterns of relationships between firms.

The evaluation of information systems and the measurement of their value to the business are two areas of increasing concern to organizations as the high levels of investment in information technology (IT) show no signs of falling. However, evaluation is a complex tangle of financial, technical and organizational threads, many of which are either avoided or dealt with ineffectively by organizations. Moreover, evaluation itself is an information intensive decision making process which requires highly heterogeneous data from a variety of sources.

Effective evaluation requires a broad understanding of the organizational context, culture and history. The evaluation process should be carried out by collaborative groups of various stakeholders, interacting through common organizational and evaluation models. The interaction between the stakeholder groups involved should be supported in terms of improved communication and negotiation capacity. 
This paper argues that considerable benefits could be gained by the provision of computerbased support in the form of an evaluation support environment which would form the focus for model development and decision-making. It could become a communication mechanism between decision makers, working mainly as a group, as well as improving the accuracy and speed of the evaluation process and allowing a wider range of data and criteria to be considered more systematically. Additionally, previous experience accumulated to a knowledge base would facilitate learning.

\section{INFORMATION SYSTEMS EVALUATION AS A GROUP DECISION PROCESS}

Information systems evaluation can clearly be viewed as a decision process where data is collected and analysed as a prelude to taking a decision regarding the development or continued operation of an information system. However, whereas in the past this process was largely monopolised by the IS department, the growing importance to organizations of both information systems and their evaluation suggests that this monopoly should come to an end. This view is supported by the increasing emphasis on organizational coordination and the growing use of cross-functional teams, as well as the shift towards viewing IS evaluation as a business function. The need to consider information systems in a broader organizational context implies the consideration and involvement of various stakeholders from different parts of the organization. The growth of interorganizational systems has meant that external trading partners, service providers and regulators need to interact together in the various stages of the evaluation process. Effective evaluation means understanding and taking seriously the perspectives of individual stakeholders and interest groups (Symons, 1991). It also means examining the mechanisms of representation of different interests, the institutional means by which divergent evaluations can be discussed, and the ability of different groups to have access to informed opinion and relevant data regarding the options available. By facilitating communication and consultation in this way, evaluation can encourage the involvement and commitment of stakeholder groups. Evaluation can thus play a central role in the process of organizational change accompanying the introduction of information systems.

\section{THE NEED FOR COMPUTER-BASED SUPPORT}

The use of computer-based tools to support decision making processes is a well established area of research and some success has been achieved in using information and decision models to narrow the gap between human ability and the requirements of a problem situation (Mumford, 1991). They are clearly of great assistance in the collection, analysis and distribution of information. Mumford (1991) also argues that IT changes the nature of decision making as it uses documentation rather than observation as its data source. While reducing the scope for human judgement and intuition, it can greatly speed-up the decision making process. It can also lead to more consistent and predictable incremental decisions. 
Computer-supported systems for data presentation, communications, data analysis and modelling appear to increase some participants' interest in the group activity. Furthermore, the transfer of some of the protocol of decision making onto technological devices may reduce intergroup tension in difficult decision situations. The technology is perceived, correctly or incorrectly, as neutral in respect to the issues discussed (Kraemer and King, 1988).

Persson (1991) approached the investment decision process as a process of handling information and knowledge. He attempted to model the process and analysed the possibilities offered by IT to improve the process. He found that a focus on the 'choice' part and capital budgeting techniques alone could not explain the manager's decisions. When the implications are strategic, social and political aspects of the decision are important. This demonstrates that technology alone has considerable limitations in a complex organizational decision situation such as investment appraisal. The tasks, culture, social structure, and individual human aspects are all essential components of the evaluation process, and unless the computer-based tool can fit seamlessly into this structure, failure is likely to result (Norman, 1991).

Interestingly, despite the extensive literature on paper-based tools and techniques for IS evaluation, very few computer-based tools have been developed that support the investment decision (project appraisal and selection) process. This situation reflects the complexity and ambiguity of IS evaluation, perhaps together with an element of the 'cobbler's children being the last to receive new shoes'. However, two knowledge-based tools have been reported in the literature, including one that helps decision makers formulate goal-programming models to select a feasible set of information systems projects (Santhanam and Schniederjans, 1993). The other system (Agarwal et al., 1992) allows users to rank projects based on qualitative and quantitative criteria. Other computer-based tools are in use but they focus on the quantitative evaluation of IT investment, supporting either finance-based activities (e.g. cost benefit analysis, ROI) or the estimation of software costs and resources (Fenton, 1991; Lederer and Prasad, 1993) (e.g. METRICS, OLIVER, SIMON, ESTIMACS, SOFTCOST, SLIM). These software tools are fairly crude, being little more than spreadsheets or automated algorithms which do not take into account the qualitative and intangible factors or the variety of stakeholders involved. Therefore, they are unable to provide a 'rich picture' of the evaluation activities as described above. Furthermore, they are unable to reflect the continuous nature of benefits and risk management.

\section{THE SUPPORT ENVIRONMENT - CONCEPTUAL FOUNDATION}

\subsection{The conceptual framework}

We argued that the elusive and complex nature of evaluating an information system is partly due to the numerous contextual factors. This requires a firm framework which can act as a foundation for discussion of the various aspects of IS evaluation in its organizational and business context. The conceptual framework discussed below aims at facilitating understanding and learning, as well as acting as a foundation for our proposed evaluation method. This framework builds on a broader conceptualisation of the linkages between the context, the content, and the process of evaluation as well as providing a historical dimension in terms of the 
accumulation of expertise and organizational learning (see also Pettigrew, 1985; Serafeimidis and Smithson, 1994 \& 1995).

\section{Context}

Context is concerned with the multi-level identification of the various systems (e.g. social, political, economic) and structures (e.g. organizational processes) within which the organization is located. Various stakeholder groups, both internal and external, should be identified together with the processes and tasks with which they are involved.

\section{Content}

The content refers to the values and criteria to be considered and what should be measured. It is here where it is particularly important to look beyond the narrow quantification of costs and benefits to an analysis of the opportunities presented by IT, together with the potential constraints and risks in its application. These include the linkage to organizational goals and a consideration of the implementation process.

\section{Process}

The process layer is concerned with the way in which evaluation is carried out (the techniques and methods used), and furthermore its relationship to IS planning, decision making, systems development and project management methods and techniques. It is very important to establish a means of communication with all the stakeholders involved in order to achieve organizational and individual learning.

\section{History}

A historical understanding of all the above conceptual elements is necessary because IT-related changes and their evaluation (including learning from failures) evolve over time and, at any particular point, present a series of constraints and opportunities shaped by the previous history.

\subsection{A comprehensive approach to information systems evaluation and benefits management}

In order to operationalise the framework and use it for practical advantage, it is necessary to 'translate' it into a set of methodological principles that is suitable for use by organizations and stakeholders responsible for IS evaluation. The approach described below is aimed at a systematic, comprehensive and open procedure for searching out the benefits and costs of an investment over the lifetime of a project, together with the associated risks. The approach is divided into five modules which are presented briefly below:

\section{Module 1 - Objectives}

This module focuses on an extensive study of the business direction of the company, expressed in terms of objectives and critical success factors, its strategy and business plans to achieve these objectives. All these are identified and formulated, bearing in mind the external and internal contextual factors which enable or constrain the organization's activities. 


\section{Module 2 - Requirements}

Specific business needs, representing the gap between the 'as-is' and 'to-be' situations, are defined as requirements, which must be traceable to the overall strategy. Various analyses can be used to help formulate these requirements, which are then prioritised in terms of information systems investment requirements. It is important, at the same time, to describe the generic costs, benefits and risks associated with investment in the particular activity area.

\section{Module 3 - Decision}

The key objective of this module is to generate a number of investment options, appraise them in various ways (financially, qualitatively, technically, socially) and select a set of options which makes best use of the resources available. It aims at using effective risk analysis/management and benefits management techniques to establish the most relevant performance measures and to consider remedial actions in order to facilitate the achievement of the desired benefits.

\section{Module 4 - Implementation}

This part recognises that, in the real world, requirements, however correctly specified initially, can be dynamic, usually as a result of external factors. Therefore, flexibility in the process of managing changing requirements is essential. A continuous monitoring of costs, benefits and risks is necessary during the project implementation.

\section{Module 5 - Delivery}

This module attempts to ensure that benefits are realised by going beyond the traditional one-off post-implementation evaluation exercise. The focus here is on performance measurement and continuous improvement through the operational life of the system. A critical part of this process is using the set of milestones and measures (defined above) that enable a company to monitor and control benefits delivery.

\section{THE SUPPORT ENVIRONMENT - OPERATIONAL ISSUES}

\subsection{The groupware component}

While the use of IT tools may be helpful for the individual evaluator, we have described IS evaluation as a group decision process and thus many of the tools and techniques currently being developed for groupware (or computer supported cooperative work - CSCW) are likely to be highly relevant in the context of IS evaluation. However, reflecting the lack of literature on the use of individual IT tools to support evaluation, there is little sign of much interest in employing groupware techniques for IS evaluation. And yet it is clear from the above discussion that IS evaluation would benefit from improved coordination and collaboration between the groups of stakeholders involved. All these 'evaluation parties' (Gregory and Jackson, 1992) with their varying characteristics may generate different evaluation contexts (Galliers, 1991; Hawgood and Land, 1988). The assumption should be that multiple stakeholders are involved in an evaluation task and each stakeholder only knows a subset of the constraints and the interests a project has to satisfy. The stakeholders should work together to carry out the evaluation, communicating their 
ideas to other stakeholders who can then refine them further. This should facilitate organizational learning (Galliers, 1991) by increasing the awareness of both risks and qualitative benefits, improving the understanding of the business processes, and highlighting any conflicting objectives within the organization. Furthermore, the stakeholders should have the facility to evaluate quickly and easily when necessary.

We have adopted the main principles of the groupware paradigm (Lockwood et al., 1993): collaboration, coordination and information sharing. In the case of IS investment evaluation, collaboration is essential because of the need to bring together various stakeholders and coordination is equally important because of the number of inter-related activities within the process modules. Some of these activities are necessarily sequential while others could be executed in parallel. However, some form of overall coordination is essential. Finally, the importance of information sharing can be seen in the need for common pools of data as well as the capability to exchange information and data. The IS evaluation activities could be carried out simultaneously by individuals and groups collaborating together and inter-relating their work through common organizational and evaluation models. This collaboration could be carried out with the participants in the same room, through electronic meeting tools (Nunamaker et al., 1991) or at a distance using tools aimed more at information sharing and communication (Malone et al., 1987).

However, particular attention would have to be paid to two aspects of any groupware tool to support IS evaluation. Firstly, due to their very different educational and professional backgrounds, the various stakeholders are likely to have very different 'frames of reference' (Orlikowski and Gash, 1994). This implies, at the very least, a flexibility in terms of representation of information and processes. Secondly, the political nature of evaluation is unlikely to disappear and, for any system to be accepted by the various stakeholders, it would have to recognise the possibility (and legitimacy) of political activity (Farbey et al, 1993). Scope for such activity could be provided by effective (inter-group) security controls.

\subsection{The case-based reasoning component}

The case-based reasoning (CBR) component that we propose assists the evaluators in deciding what and how to evaluate by identifying cases similar to the current situation as described by the evaluators to the CBR system. The case library would be separated into five smaller sets corresponding to each stage of the above method which would provide better recommendations to stakeholders involved at different stages of the project. However, the logical connections between each stage and the following one means that the output of each stage can be used as input to the next stage.

We argued above that IS evaluation is an information intensive activity which would benefit from computer-based support and this is particularly the case for (necessarily) complex IS projects and/or comprehensive evaluation and benefits management guidelines such as those presented above. However, while the need for computer-based support is fairly clear, we do not recommend a traditional decision support approach as conventional decision support systems have only really found favour in relatively straightforward, quantitative and repetitive decision making. Evaluation has some quantitative dimensions but it is typically the qualitative and subjective aspects that are important and thus we would recommend an approach that can 
support problem exploration and sense-making. Such an approach would seem to be offered by case-based reasoning.

Case-based reasoning is a developing research area within the field of cognitive science and artificial intelligence (Kolodner, 1993; Riesbeck and Schank, 1989). Case-based reasoning systems provide recommended solutions to a new problem by locating similar problems and their solutions in a case library. In contrast to rule-based problem solving systems which depend on the identification of problem solving heuristics, CBR systems depend on the presentation of previous experience in a suitable framework for providing recommendations to slightly different problem situations. The CBR system assists in the problem solving process either by retrieving similar cases, or by adapting the closest solution in light of the different problem situation. However, increasing numbers of applications, especially those related to manufacturing and information retrieval are being developed using CBR (Allen, 1994; Hammond, 1989; Kolodner, 1988).

Certain types of problems have eluded a clear formulation, but a considerable amount of experience has been accumulated anyway because they are problems which practitioners encounter on a regular basis. CBR systems can provide support for such problem solving and decision making where theories, models, and methods of measurement are lacking, or where there are no generally acknowledged rules, procedures, or formulae for deriving a solution.

The evaluation of an IS project appears to be one such type of problem. Numerous contextual factors affect the evaluation of IS projects, so that each problem appears to be unique, and evaluating each project is like solving a slightly different problem. Because there are so many specific features of each project to be attended to, issues of what to measure, how to measure, and why it is to be measured are not always explicit. The ability to refer to previous projects could provide the historical dimension needed for IS evaluation and the ability to refer to related projects from other organizations would be helpful. A computerised support system based on CBR technology should be able to assist in building a better understanding of the evaluation context, the selection of an appropriate evaluation method (process), and the selection of metrics to use (content) due to its ability to handle multiple criteria and large numbers of previous cases. CBR technology can process large numbers of factors that humans have difficulty grasping at the same time and it supports human opinion with previous experience embedded inside real cases. The experience preserved in the case library, once it is in place, can then be accessed and shared even amongst non-experts (Curet, 1995; Price and Pegler, 1995a \& 1995b).

\section{CONCLUSION}

This paper investigated the complicated and group-driven process of information systems evaluation. It proposed a support environment built upon a conceptual framework and a set of methodological guidelines related to investment appraisal and benefits management. The proposed system has two major components: a groupware part and a case-based reasoning part. The groupware component assists with the collaboration and communication between the multiple stakeholders involved in evaluation. The CBR component assists an evaluator in deciding what and how to evaluate by identifying cases similar to the current situation as described by the evaluator to the CBR system. 


\section{REFERENCES}

Agarwal, R., Tanniru, M.R. and Dacruz, M. (1992) Knowledge-Based Support for Combining Qualitative and Quantitative Judgements. Journal of Management Information Systems, 9 (1), 165-184.

Allen, B.P. (1994) Case-Based Reasoning: Business Applications. Communications of the ACM, 37 (3), 40-42.

Curet, O. (1995) The application of case-based reasoning to assist accountants in identifying top management fraud: a study of the problem domain and the methodological issues in the development, implementation and evaluation of a case-based learning and reasoning tool, in BCS Colloquium: Case-based reasoning: prospects for applications. Professional group C4(AI), London.

Farbey, B., Land, F. and Targett, D. (1993) How to assess your IT Investment - A study of methods and practice. Butterworth-Heinemann, Oxford.

Fenton, N.E. (1991) Software Metrics. A Rigorous Approach. Chapman \& Hall, London

Galliers, R.D. (1991) Strategic information systems planning: myths, reality and guidelines for successful implementation. European Journal of Information Systems, 1 (1), 55-64.

Gregory, A.J. and Jackson, M.C. (1992) Evaluation Methodologies: A System for Use. Journal of Operational Research Society, 43 (1), 19-28.

Hammond, K. (1989) Proceedings of the Second Case Based Reasoning Workshop (DARPA). Morgan Kaufmann Publishers, Inc., San Mateo, California.

Hawgood, J. and Land, F. (1988) A Multivalent Approach to Information Systems Assessment, in Information Systems Assessment: Issues and Challenges (eds. N. Bjorn-Andersen and G.B. Davis), North Holland, Amsterdam, 103-124.

Kolodner, J.L. (1993) Case-based reasoning. Morgan Kaufmann Publishers, Inc., San Mateo, California.

Kraemer, K.L. and King, J.L. (1988) Computer-Based Systems for Cooperative Work and Group Decision Making. ACM Computing Surveys, 20 (2), 115-146.

Lederer, A.L. and Prasad, J. (1993) Information systems software cost estimating: a current assessment. Journal of Information Technology, 8, 22-33.

Lockwood, R., Lavery, M. and Lachal, L. (1993) Groupware: Market Strategies. Ovum Limited, London.

Malone, T.W., Grant, K.R., Turbak, F.A., Brobst, S.A. and Cohen, M.D. (1987) Intelligent information-sharing systems. Communications of the ACM, 30 (5), 390-402.

Mumford, E. (1991) Decision Making and the Organizational Environment: Today's Problems and Tomorrow's Needs, in Environments for Supporting Decision Processes (eds. H.G. Sol and J. Vescenyi), North Holland, Amsterdam, 1-12.

Norman, D.A. (1991) Collaborative Computing: Collaboration First, Computing Second. Communications of the $A C M, 34$ (12), 88-90.

Nunamaker, J.F., Dennis, A.R., Valacich, J.S., Vogel, D.R. and George, J.F. (1991) Electronic meeting systems to support group work. Communications of the ACM 34 (7), 40-6.

Orlikowski, W. and Gash, D.C. (1994) Technological Frames: Making Sense of Information Technology in Organizations, ACM Transactions on Information Systems, 12 (2), 174-207. 
Persson, I. 1991. The use of information technology in the investment process - An Analysis of some possibilities, in Environments for Supporting Decision Processes (eds. H.G. Sol and J. Vescenyi), North Holland, Amsterdam, 239-255.

Pettigrew, A. (1985) The Awakening Giant: Continuity and Change in ICI. Blackwell, Oxford.

Price, C. and Pegler, I.S. (1995a) Deciding parameter values with case-based reasoning, in Proceedings of the First UK Case-based reasoning workshop (ed. I. Watson), Salford.

Price, C. and Pegler, I.S. (1995b) Wayland: Efficiency improvements in aluminium die casting, in BCS Colloquium: Case-based reasoning: prospects for applications, Professional group C4(AI), London.

Riesbeck, C.K. and Schank, R.C. (1989) Inside case-based reasoning. L. Erlbaum, Hillsdale, New Jersey.

Santhanam, R. and Schniederjans, M.J. (1993) A Model Formulation System for Information Systems Project Selection. Computers and Operational Research, 20 (7), 679-690.

Serafeimidis, V. and Smithson, S. (1994) Evaluation of IS/IT Investments: Understanding and Support, in Proceedings of The First European Conference on Information Technology Investment Evaluation, (eds. A. Brown and D. Remenyi), Henley Management College, England.

Serafeimidis, V. and Smithson, S. (1995) The management of change for a rigorous appraisal of IT investment. The case of a UK insurance organization, in Proceedings of the 3rd European Conference on Information Systems, (ed. G.I. Doukidis), Athens, Greece.

Symons, V.J. (1991) A review of information systems evaluation: content, context and process. European Journal of Information Systems, 1 (3), 205-212.

\section{BIOGRAPHIES}

Vasilis Serafeimidis, is a graduate of the Athens University of Economics and Business. He also holds an MSc degree in Analysis, Design and Management of the Information Systems. He has worked as a consultant for a number of years and currently he is a $\mathrm{PhD}$ candidate and a teaching assistant at the London School of Economics (University of London). His major research interests are the appraisal of IT investments, risk management of IT projects, decision support environments.

Steve Smithson, is a senior lecturer in information systems at the London School of Economics. $\mathrm{He}$ holds a BSc and MSc both from the London School of Economics. He received his $\mathrm{PhD}$ in Information Systems in 1989. Previously, he worked for twelve years in industry, mostly in transport and distribution. His main research interests are in information systems management and the application of IT into organizations. He is also editor of the European Journal of Information Systems. 